\title{
Does diet quality or nutrient quantity contribute more to health?
}

\author{
Justin B. Echouffo-Tcheugui and Rexford S. Ahima
}

Division of Endocrinology, Diabetes \& Metabolism, Department of Medicine, Johns Hopkins University School of Medicine, Baltimore, Maryland, USA.

\section{Diet and disease risk}

Western diet is a leading risk factor for chronic illness and death (1). A recent study in the journal Lancet addressed key questions pertaining to the health-related effects of nutrition as assessed through the dietary components (2). The Global Burden of Diseases, Injuries, and Risk Factors Study (GBD) 2017 Diet Collaborators evaluated 15 dietary factors for their effects on mortality and disability from cardiovascular diseases, diabetes, and cancer across 195 countries (2). Adding to their previous findings (3), the GBD study made a number of key observations. First, the consumption of healthy diet was suboptimal worldwide (i.e., low intake of nuts and seeds, milk, and whole grains), whereas the consumption of unhealthy diet (i.e., sugary beverages, salt, and processed and red meat) was more common. Second, the examined dietary factors were potentially responsible for a high disease burden, including 11 million (95\% uncertainty interval, 10-12) deaths and 255 million (95\% uncertainty interval, 234-274) disability-adjusted life-years (DALYs; $22 \%$ of all deaths and $15 \%$ of all DALYs in adults aged 25 years or older). Third, three factors, including high intake of sodium, low intake of whole grains, and low intake of fruit, accounted for more than $50 \%$ of diet-related deaths and two-thirds of diet-related DALYs. These findings were consistent across different regions of the world.

\section{Dietary patterns \\ versus nutrient-based \\ recommendations}

The findings of the GBD group add to growing evidence supporting the importance of diet quality defined by dietary patterns, which appear to have a greater effect on health outcomes compared with

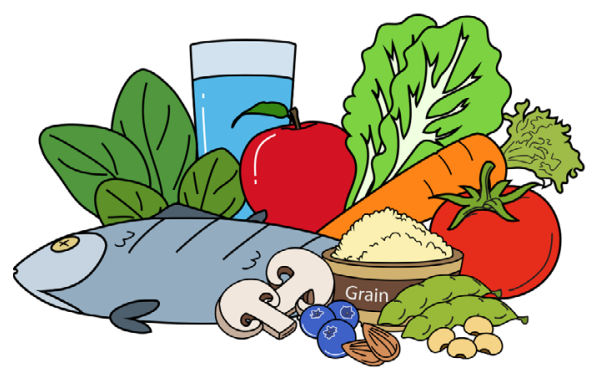

Healthy diet

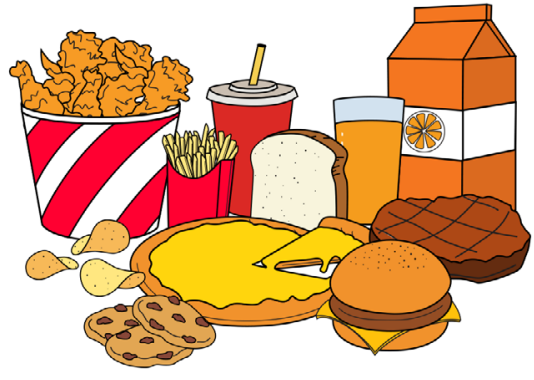

Unhealthy diet
Figure 1. Representative healthy and unhealthy quality diets. A healthy quality diet includes high intake of fruits, nuts and seeds, vegetables, fish, legumes, and cereals and limited intake of meat and dairy products, whereas sugary beverages, high salt, and processed and red meat are indicative of an unhealthy quality diet. Illustrated by Mao Miyamoto. individual dietary components or nutrients. The growing evidence endorses a shift from a nutrient-based to a dietary pattern (food-based) approach in nutritional guidelines. Indeed, it is logical to use a dietary pattern approach instead of nutrient quantity for disease management and prevention. Dietary patterns represent a combination of foods (which deliver nutrients) habitually consumed by an individual (Figure 1). Dietary patterns are conceptually more appropriate for disease management and prevention for a number of reasons. One argument is that, for various dietary factors and total energy intake involved in disease processes, there are interactions across foods, and dietary patterns are more translatable to dietary advice, i.e., "people eat foods, not individual nutrients"(4). Another argument for adopting a dietary pattern approach is the inconsistent clinical benefit of diets focused on isolated nutrient components. For example, low-fat and low-saturated-fat diets do not consistently exert benefits on cardiovascular diseases or diabetes mellitus in both observational studies
Conflict of interest: The authors have declared that no conflict of interest exists. and randomized clinical trials (5-7). This contrasts sharply with findings from clinical trials evaluating the effects of dietary patterns on health outcomes (8-11).

\section{Findings from clinical studies}

The most-studied healthy dietary patterns are the Mediterranean and Dietary Approaches to Stop Hypertension (DASH) diets. Mediterranean diet refers to a diet encouraging high intake of fruits, nuts and seeds, vegetables, fish, legumes, and cereals and limited intake of meat and dairy products (4). Mediterranean diet has been associated with favorable health outcomes in both observational and experimental studies. In prospective cohort studies, Mediterranean diet has been associated with lower risk for type 2 diabetes, cardiovascular disease, and cancer (4). A randomized controlled clinical trial of Mediterranean diet versus control, the Prevención con Dieta Mediterránea (PREDIMED) study (8-10), showed a 30\% reduction in cardiovascular events in the intervention group. Secondary analyses in the PREDIMED study showed benefits in terms of reduction in the incidence of type 2 diabetes (9) and breast cancer (11).

The DASH diet promotes consumption of vegetables and fruits, lean meat, and dairy products; inclusion of micro- 
nutrients in the diet; reduction of sodium in the diet to about $1500 \mathrm{mg} / \mathrm{d}$; and consumption of minimally processed and fresh food (4). The DASH diet pattern has been shown to improve a wide spectrum of disease risk factors, including hypertension and long-term weight reduction, and is consistently associated with lower risks of clinical events (12-14). Moreover, a modified DASH diet, which is additionally higher in vegetable fats and lower in carbohydrates, similar to Mediterranean diet, has been shown to improve cardiometabolic outcomes $(15,16)$. Other studies of dietary patterns similar to Mediterranean or DASH diets have also shown benefits for cardiovascular diseases, diabetes, obesity, and cancer (4). Given the vast sociocultural and economic differences across the world, not every population can consume a traditional Mediterranean diet; however, the adoption of Mediterranean-style dietary patterns in various regions of the world is generally feasible (17).

In contrast to a Western-style diet, the beneficial action of healthy quality diets, such as the Mediterranean or DASH diet, encompasses effects on blood pressure, insulin sensitivity, lipids, inflammation, oxidative stress, endothelial function, coagulation, and the gut microbiome (18). It is important to point out that, in largescale epidemiological studies and clinical trials, the adoption of healthy dietary patterns was able to mitigate the genetic risk of weight gain, with a significantly greater effect of healthy diet quality on weight in people with high genetic risk for obesity (19). In addition, there is evidence linking maternal consumption of Mediterranean diet to specific differentially methylated DNA regions in the infant (20).

\section{Conclusions}

In summary, different lines of investigation suggest that "dietary quality" rather than "nutrient quantity" is a more reliable index for healthy nutrition. Any meaningful dietary intervention ought to extend far beyond the current focus on nutrient quantity and include the myriad qualitative aspects of food and food combinations, which would affect health and disease states. Given the enormous human and economic toll of diseases related to unhealthy diets, we are proposing that it is time for nutritional scientists to develop measurable and reliable dietary quality indicators that could be used for nutrition guidelines across diverse regions of the world.

\section{Acknowledgments}

RSA is supported by NIH grants R01NS084965 and RF1AG059621 and a Bloomberg Distinguished Professorship.

Address correspondence to: Justin B. Echouffo-Tcheugui and Rexford S. Ahima, Department of Medicine, Division of Endocrinology, Diabetes \& Metabolism, Johns Hopkins University School of Medicine, 1830 East Monument Street, Baltimore, Maryland 21287, USA. Phone: 410.550.3054; Email: jechouf1@jhmi. edu (JBET). Phone: 410.550.7626; Email: ahima@jhmi.edu (RSA).

1. Lim SS, et al. A comparative risk assessment of burden of disease and injury attributable to 67 risk factors and risk factor clusters in 21 regions, 1990-2010: a systematic analysis for the Global Burden of Disease Study 2010. Lancet. 2012;380(9859):2224-2260.

2. GBD 2017 Diet Collaborators. Health effects of dietary risks in 195 countries, 19902017: a systematic analysis for the Global Burden of Disease Study 2017. Lancet. 2019;393(10184):1958-1972.

3. GBD 2017 Risk Factor Collaborators. Global, regional, and national comparative risk assessment of 84 behavioural, environmental and occupational, and metabolic risks or clusters of risks for 195 countries and territories, 1990-2017: a systematic analysis for the Global Burden of Disease Study 2017. Lancet. 2018;392(10159):1923-1994.

4. Schulze MB, Martínez-González MA, Fung TT, Lichtenstein AH, Forouhi NG. Food based dietary patterns and chronic disease prevention. BMJ. 2018;361:k2396.

5. Howard BV, et al. Low-fat dietary pattern and risk of cardiovascular disease: the Women's Health Initiative Randomized Controlled Dietary Modification Trial. JAMA. 2006;295(6):655-666.

6. Tinker LF, et al. Low-fat dietary pattern and risk of treated diabetes mellitus in postmenopausal women: the Women's Health Initiative randomized controlled dietary modification trial. Arch Intern Med. 2008;168(14):1500-1511.

7. Micha R, Mozaffarian D. Saturated fat and cardiometabolic risk factors, coronary heart disease, stroke, and diabetes: a fresh look at the evidence. Lipids. 2010;45(10):893-905

8. Estruch R, et al. Primary prevention of cardiovascular disease with a Mediterranean diet. N Engl J Med. 2013;368(14):1279-1290.

9. Salas-Salvadó J, et al. Prevention of diabetes with Mediterranean diets: a subgroup analysis of a randomized trial. Ann Intern Med. 2014;160(1):1-10.

10. Estruch R, et al. Primary prevention of cardiovascular disease with a mediterranean diet supplemented with extra-virgin olive oil or nuts. N Engl J Med. 2018;378(25):e34.

11. Toledo E, et al. Mediterranean Diet and Invasive Breast Cancer Risk Among Women at High Cardiovascular Risk in the PREDIMED Trial: A Randomized Clinical Trial. JAMA Intern Med. 2015;175(11):1752-1760.

12. Appel LJ, et al. A clinical trial of the effects of dietary patterns on blood pressure. DASH Collaborative Research Group. N Engl J Med. 1997;336(16):1117-1124.

13. Fung TT, Chiuve SE, McCullough ML, Rexrode KM, Logroscino G, Hu FB. Adherence to a DASH-style diet and risk of coronary heart disease and stroke in women. Arch Intern Med. 2008;168(7):713-720.

14. Salehi-Abargouei A, Maghsoudi Z, Shirani F, Azadbakht L. Effects of Dietary Approaches to Stop Hypertension (DASH)-style diet on fatal or nonfatal cardiovascular diseases--incidence: a systematic review and meta-analysis on observational prospective studies. Nutrition. 2013;29(4):611-618

15. Appel LJ, et al. Effects of protein, monounsaturated fat, and carbohydrate intake on blood pressure and serum lipids: results of the OmniHeart randomized trial. JAMA 2005;294(19):2455-2464.

16. Gadgil MD, Appel LJ, Yeung E, Anderson CA Sacks FM, Miller ER. The effects of carbohydrate, unsaturated fat, and protein intake on measures of insulin sensitivity: results from the OmniHeart trial. Diabetes Care. 2013;36(5):1132-1137.

17. Anand SS, et al. Food Consumption and its Impact on Cardiovascular Disease: Importance of Solutions Focused on the Globalized Food System: A Report From the Workshop Convened by the World Heart Federation. J Am Coll Cardiol. 2015;66(14):1590-1614.

18. Lou-Bonafonte JM, Gabás-Rivera C, Navarro MA, Osada J. PON1 and Mediterranean Diet. Nutrients. 2015;7(6):4068-4092.

19. Wang $\mathrm{T}$, et al. Improving adherence to healthy dietary patterns, genetic risk, and long term weight gain: gene-diet interaction analysis in two prospective cohort studies. BMJ. 2018;360:j5644.

20. House JS, et al. Periconceptional maternal mediterranean diet is associated with favorable offspring behaviors and altered $\mathrm{CpG}$ methylation of imprinted genes. Front Cell Dev Biol. 2018;6:107. 